\title{
MicroRNAs and Lymph Node Metastasis in Papillary Thyroid Cancers
}

\author{
Nurul-Syakima Ab Mutalib ${ }^{1 * \&}$, Azliana Mohamad Yusof ${ }^{1 \&}$, Norfilza Mohd \\ Mokhtar $^{2}$, Roslan Harun ${ }^{3}$, Rohaizak Muhammad ${ }^{4}$, Rahman Jamal $^{1}$
}

\begin{abstract}
Lymph node metastasis (LNM) in papillary thyroid cancer (PTC) has been shown to be associated with increased risk of locoregional recurrence, poor prognosis and decreased survival, especially in older patients. Hence, there is a need for a reliable biomarker for the prediction of LNM in this cancer. MicroRNAs (miRNAs) are small noncoding RNAs that regulate gene translation or degradation and play key roles in numerous cellular functions including cell-cycle regulation, differentiation, apoptosis, invasion and migration. Various studies have demonstrated deregulation of miRNA levels in many diseases including cancers. While a large number of miRNAs have been identified from PTCs using various means, association of miRNAs with LNM in such cases is still controversial. Furthermore, studies linking most of the identified miRNAs to the mechanism of LNM have not been well documented. The aim of this review is to update readers on the current knowledge of miRNAs in relation to LNM in PTC.
\end{abstract}

Keywords: microRNAs - papillary thyroid cancer - lymph node - metastasis - biomarker

Asian Pac J Cancer Prev, 17 (1), 25-35

\section{Introduction}

Papillary thyroid carcinoma (PTC) is the commonest type of thyroid cancer, contributing to more than $80 \%$ of all thyroid malignancies. Generally, the prognosis of patients with PTC is relatively good compared to other cancers, with an overall 10-year survival rate of more than 90\% (Lee et al., 2013).

The global incidence of PTC has dramatically increased in the past decade and its epidemiology is changing (Vigneri et al., 2015). Furthermore, certain clinicopathologic features have been associated with poorer prognosis, such as older age at diagnosis (Hsieh et al., 2012), gender (Jonklaas et al., 2012), large primary tumor ( $\geq 2 \mathrm{~cm}$ ) (Kramer et al., 2010), extrathyroidal invasion (Hotomi et al., 2012), BRAF V600E mutation status (Xing et al., 2013), multifocality (Qu et al., 2014) and lymph node metastasis (LNM) (Zaydfudim et al., 2008; Lee et al., 2014). In addition, several studies have demonstrated that the presence of LNM is associated with locoregional recurrence and with an increased risk of mortality prominently among older patients (Scheumann et al., 1994; Machens et al. 2002; Lundgren et al., 2006).

Despite the excellent prognosis, the major challenge in PTC involves controlling locoregional recurrence and hence the reason lymph node surgery is considered important in the treatment of PTC (Moo and Fahey, 2011).

MicroRNAs (miRNAs), firstly identified in Caenorhabditis elegans, are endogenous, non-proteincoding single-stranded RNAs containing between 19-24 nucleotides and are derived from a stem-loop precursor to regulate gene expression by binding primarily to the 3'-UTR of specific 'target' messenger RNA (mRNAs). These non-protein-coding RNAs (together with other intronic RNAs) constitute the majority of genomic output in complex eukaryotes (Mattick, 2001). MiRNAs that bind with perfect or nearly perfect complementarity to proteincoding mRNA sequences will induce the RNA-mediated interference (RNAi) pathway, resulting in the disruption of mRNA stability and/or translation (Bartel, 2009). Due to their post-transcriptional regulatory effects, the key function of miRNAs is to 'fine tune' the level of proteins involved in numerous biological processes, including embryogenesis, organogenesis, tissue homeostasis, immune system function and cell cycle control (Nam et al., 2009).

Owing to its ability to form partially perfect complimentary binding with the target genes, a single miRNA is able to regulate the expression of more than 100 different transcripts. It has been estimated that these

${ }^{1}$ UKM Medical Molecular Biology Institute (UMBI), Cheras, ${ }^{2}$ Department of Physiology, ${ }^{4}$ Department of Surgery, Faculty of Medicine, Universiti Kebangsaan Malaysia, Kuala Lumpur, ${ }^{3}$ Suite 4-4, KPJ Ampang Puteri Specialist Hospital, Selangor, Malaysia ${ }^{\circledR}$ Equal contributors*For correspondence: nurulsyakima@gmail.com / syakima@ppukm.ukm.edu.my 
molecules may be able to regulate up to $30 \%$ of the protein-coding genes in the human genome (Felekkis, 2010), resulting in increased widespread attention on their potential role in complex biological processes and heterogenous diseases. To date there are 1,881 precursors and 2,588 mature miRNA genes which have been described in the human genome. The latest miRBase release contains 24,521 miRNA loci from 206 species, which can be processed to produce 30,424 mature miRNA products (Kozomara and Griffiths-Jones, 2014).

Dysregulation of miRNAs expression in human cancers have been demonstrated by many studies (Iorio and Croce, 2012). Through expression profiling studies, miRNA was shown to be linked to tumor development, progression as well as response to treatment, signifying their potential use as biomarker for diagnosis and prognosis (Iorio and Croce, 2012). The involvement of these molecules in human cancers can be explained by the fact that more than $50 \%$ of miRNA genes are located in cancer-associated genomic regions or in fragile sites, minimal regions of loss of heterozygosity, minimal regions of amplification, or common breakpoint regions (Calin et al., 2004). Additionally, miRNAs are strongly conserved among different species, further adding the value of their important roles in many crucial biological processes (Marini et al., 2011).

Clinically detectable lymph node metastasis (LNM) occurs in $15 \%$ to $30 \%$ of PTC cases (Shaha et al., 1996; Wada et al., 2003). It is well established that PTC patients with LNM have an increased risk of recurrence and mortality (Guerrero and Clark, 2011). Even though this condition may not be immediately fatal, it poses a great challenge to the oncologists and stressful to the patients (Ito et al., 2012). PTC patients with LNM at initial presentation have a higher incidence of recurrent disease in the cervical region (Cognetti et al., 2008) and approximately a ten-fold increased risk of developing a nodal recurrence (McConahey et al., 1986). Several predictive factors for LNM have been established, including age, gender, tumor size and histopathologic characteristics of the tumor. In addition, miRNAs have also been shown to be potential biomarkers in predicting LNM. Several miRNAs was proven to be associated with LNM; there was a positive correlation between high miR-21 expression and tumor stage and LNM in patients with breast cancer (Yan et al., 2008), and development of distant metastases in colorectal cancer patients (Slaby et al., 2007). Most recently, miR-1207-5p was suggested as a useful biomarker in the prediction of LNM in gastric cancer (Huang et al., 2015).

To date, studies focusing on the involvement of miRNAs in PTC with lymph node metastasis are quite limited (Table 1). A literature search using PubMed/ MEDLINE, ScienceDirect, Scopus and Google Scholar revealed 16 miRNAs potentially involved in PTC with LNM of which nine were upregulated and eight were downregulated (Figure 1). Majority of miRNA studies in PTC focused on comparing thyroid malignancies (including PTC) to normal thyroid tissues or benign thyroid diseases such goiter (He et al., 2005; Pallante et al., 2006; Tetzlaff et al., 2007; Chen et al., 2008; Nikiforova et al., 2008; Yip et al., 2011; Dettmer et al., 2013; The Cancer Genome Atlas Research Network, 2014). Reviews on thyroid cancer have also highlighted the utility of miRNAs in distinguishing malignant from benign lesions (Pallante et al., 2010; Braun and Hüttelmaier, 2011; de la Chapelle and Jazdzewski, 2011; Marini et al., 2011; Leonardi et al., 2012; Lodewijk et al., 2012; Li et al., 2013; Samimi et al., 2013; Pallante et al., 2014). On the other hand, a review on the significance of the miRNAs in PTC with and without LNM is lacking (Yuan et al., 2014). Hence, this review aims to report the extent of involvement of miRNAs in PTCs with LNM, and to discuss the potential clinical significance of the miRNAs in patients with PTC.

\section{miRNAs Upregulated in PTC with LNM}

miR-136

miR-136, located on chromosome 14 , has been shown to be upregulated in the Jurkat cell line (Yu et al., 2006) and prominently overexpressed in murine lung cancers (Liu et al., 2010). It has also been shown to target the tumor suppressor PTEN by repressing its translation (Lee et al., 2010), which strongly suggests its role in cancer development and/or progression. A more recent study has strengthened further the evidence on the role of miR-136 in cancer, whereby the suppression of miR-136 expression in a non-small cell lung cancer cell line inhibited both anchorage-dependent and anchorage-independent proliferation (Shen et al., 2014). With regards to PTC, Peng and colleagues reported miR-136-5p upregulation in an aggressive phenotype of the cancer (Peng et al., 2014). The biological function of miR-136 in regulating LNM in PTC remains largely unknown. Anchorage-independent proliferation is one of the hallmarks of cancer, mainly pertaining to metastatic potential. Since this miRNA was shown to have the ability to elicit anchorage-independent growth in vitro in lung cancer, the involvement of miR136 in metastasis process in PTC is worth investigating.

\section{miR-146}

miR-146 is one of the widely studied miRNAs in thyroid cancers and has been shown to be frequently upregulated in PTC (He et al., 2005; Pallante et al., 2006; Tetzlaff et al., 2007; Chen et al., 2008; Chou et al., 2010; Yip et al., 2011; Chou et al., 2013; Sun et al., 2013a), anaplastic thyroid cancer (Fassina et al., 2014) and follicular thyroid cancer (FTC) (Wojtas et al., 2014).

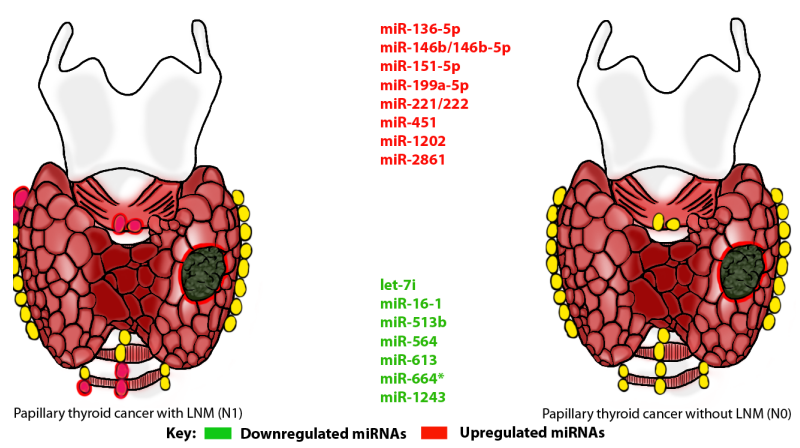

Figure 1. miRNAs implicated in regulation of lymph node metastasis in PTC. 
Table 1. miRNAs significantly differentially expressed in PTC with LNM versus without LNM

\begin{tabular}{|c|c|c|c|}
\hline \multirow{2}{*}{ References } & \multicolumn{2}{|c|}{ (LNM vs. without LNM) } & \multirow{2}{*}{ Detection method } \\
\hline & Upregulated miRNAs & Downregulated miRNAs & \\
\hline \multirow{2}{*}{$\begin{array}{l}\text { Lee et al., } \\
\qquad 2013\end{array}$} & miR-146b & \multirow{2}{*}{ NA } & \multirow{2}{*}{ Real time PCR } \\
\hline & miR-222 & & \\
\hline \multirow{2}{*}{$\begin{array}{l}\text { Peng et al., } \\
\quad 2014\end{array}$} & miR-136-5p & $\operatorname{miR}-513 b$ & \multirow{2}{*}{$\begin{array}{l}\text { Microarray (Exiqon miRCURY LNA chip } \\
\text { corresponding to miRBASE release 16) }\end{array}$} \\
\hline & miR-199a-5p & $\mathrm{miR}-1243$ & \\
\hline \multirow{2}{*}{$\begin{array}{l}\text { Sun et al., } \\
2013 \mathrm{a}\end{array}$} & miR-221 & \multirow{2}{*}{ NA } & \multirow{2}{*}{ Real time PCR } \\
\hline & miR-222 & & \\
\hline \multirow{3}{*}{$\begin{array}{l}\text { Yang et al., } \\
\quad 2013\end{array}$} & miR-146b-5p & miR-16-1 & \multirow{3}{*}{$\begin{array}{l}\text { Microarray (Affymetrix }{ }^{\circledR} \text { GeneChip miRNA } 2.0 \\
\text { array containing } 1,105 \text { known human miRNAs) }\end{array}$} \\
\hline & miR-221 & $\mathrm{miR}-613$ & \\
\hline & miR-222 & mIR-015 & \\
\hline \multirow{3}{*}{$\begin{array}{l}\text { Acibucu et } \\
\text { al., } 2014\end{array}$} & $\mathrm{miR}-146 \mathrm{~b}$ & \multirow{3}{*}{ NA } & \multirow{3}{*}{ Real time PCR } \\
\hline & miR-221 & & \\
\hline & miR-222 & & \\
\hline $\begin{array}{l}\text { Deng et al., } \\
2014\end{array}$ & miR-146b-5p & NA & Real time PCR \\
\hline \multirow{2}{*}{$\begin{array}{l}\text { Yu et al., } \\
2012\end{array}$} & $\operatorname{miR}-151-5 p$ & \multirow{2}{*}{ NA } & \multirow{2}{*}{ Sequencing (Solexa) } \\
\hline & miR-222 & & \\
\hline \multirow{4}{*}{$\begin{array}{l}\text { Wang et al., } \\
\text { 2013c }\end{array}$} & miR-451 & let-7i & \multirow{4}{*}{$\begin{array}{l}\text { Microarray (Agilent Human miRNA array containing } \\
\qquad 1,205 \text { human miRNA sequences) }\end{array}$} \\
\hline & miR-1202 & miR-542-5p & \\
\hline & miR-2861 & miR-564 & \\
\hline & & miR-664* & \\
\hline
\end{tabular}

In addition, PTC with LNM demonstrated significantly higher expression of miR-146b as compared to cases without LNM, suggesting its role in metastasis (Yang et al., 2013; Acibucu et al., 2014; Deng et al., 2015). Functional analyses of miR-146 revealed its involvement in migration, invasion, proliferation and cell cycle (Geraldo et al., 2012; Chou et al., 2013; Deng et al., 2015).

Bioinformatics screening and in silico target prediction using TargetScan (http://www.targetscan.org) has revealed $S M A D 4$, an important member of the transforming growth factor $\beta$ (TGF- $\beta$ ) signaling pathway, as the potential target of miR-146b-5p (Geraldo et al., 2012). The authors firstly confirmed the direct binding of miR-146b-5p on the SMAD4 UTR via the luciferase reporter assay, and followed this with the inhibition of miR-146b-5p using a locked nucleic acid inhibitor. As expected, the inhibition resulted in significantly increased SMAD4 gene and protein levels in the human PTC cell lines. Furthermore, the inhibition of miR-146b-5p increased the cellular response to the TGF- $\beta$ anti-proliferative signal, leading to significant reduction of cell proliferation. On the contrary, overexpression of miR-146b-5p in normal rat follicular cells reduced SMAD4 levels, interrupted TGF- $\beta$ signal transduction, conferred resistance to TGF- $\beta$-mediated cellcycle arrest and significantly increased cell proliferation (Geraldo et al., 2012).

Using multivariate logistic regression analysis, Chou and colleagues demonstrated that miR-146b expression is one of the independent risk factors for poor prognosis in PTC, implicating the potential of this miRNA as a prognostic marker (Chou et al., 2013). Patients with higher miR-146b expression levels had significantly worse overall survival compared to patients with lower miR-146b levels (Chou et al., 2013). Functional characterization revealed that transient overexpression of miR-146b significantly increased cell migration, invasion, colony-forming ability and most importantly, conferred resistance to chemotherapy-induced apoptosis in BRAFmutated cell lines (Chou et al., 2013).

In a more recent study, expression analysis using real time polymerase chain reaction (qPCR) in 60 primary PTC patients with and without LNM revealed upregulation of miR-146b-5p and downregulation of Zinc Ring Finger 3 (ZNRF3) gene (Deng et al., 2015). Luciferase assay confirmed ZNRF3 as a direct target of miR-146b-5p and this miRNA was shown to stimulate cell migration, invasion and epithelial-to-mesenchymal transition (EMT) by downregulating ZNRF3 (Deng et al., 2015). Another study showed that ZNRF3 inhibits Wnt signaling by interacting with FZD and LRP 5/6 complexes, and promotes Wnt receptor ubiquitination and degradation (Hao et al., 2012). miR-146b-5p increases the cell surface levels of FZD6 and LRP6 via suppression of ZNRF3, causing enhanced $\mathrm{Wnt} / \beta$-catenin signaling. These findings divulged a novel mechanism of miR-146b-5p mediated induction of EMT and implied the role of ZNRF3 as a tumor suppressor in PTC (Deng et al., 2015).

On the other hand, another study performed in 91 PTC patients failed to find any significant association of miR-146b with LNM (Wang et al., 2013). The reason for this remains unclear and it might be contributed by the use of a different platform and/or chemistry for detection 
and also possibly the intrinsic variability in the cohort of patients. In addition, even though the tissues used in the studies were verified by a pathologist, the authors did not mention about the percentage of cancer cells. Perhaps a more refined quality control and assessment of the tumour specimens before being subjected to expression profiling will be able to explain such contradictory findings.

miR-151

MicroRNA-151 (miR-151), a frequently amplified miRNA localized to chromosome 8 at q24.3, can concurrently express two mature sequences: miR-151-3p and miR-151-5p (Ding et al., 2010). Genome-wide serum miRNA expression profiling using Solexa sequencing followed by extensive validation in 245 subjects reveals upregulation of miR-151-5p in PTC patients (Yu et al., 2012). Elevated levels of miR-151-5p in circulation and the tissues of PTC patients compared with benign cases and healthy controls prompted further assessment of the relationship between this miRNA and clinicopathological features including gender, age, tumor size, multifocality, LNM, TNM stages and BRAF mutation status. The serum levels of miR-151-5p was significantly higher in lymph node-positive patients than in lymph node-negative patients (Yu et al., 2012), suggesting potential utility of this miRNA in predicting LNM. In addition, overexpression of serum miR-151-5p was strongly associated with tumor size $(\mathrm{P}<0.001)$.

The opposite observation of miR-151 levels has been reported by other cancer studies. In a cohort of breast cancer patients, miR-151-5p expression was significantly lower in the lymph-node metastases than in their corresponding tumors (Krell et al., 2012). The authors suggested that miR-151-5p upregulation may suppress metastasis in primary breast tumors (Krell et al., 2012). Given a limited knowledge available regarding functional role of miR-151-5p and the contradictory findings in different cancer types, further investigation of the role of miR-151 in LNM in thyroid cancer is worth pursuing.

\section{miR-199}

miR-199b-5p has been reported to be downregulated in follicular thyroid carcinoma and a gain-of-function experiment in the same study showed reduced cell growth (Rossing et al., 2012). It also has a positive correlation with PTC invasiveness and BRAF V600E mutation (Chou et al., 2010). In a more recent research, Peng and colleagues reported that miR-199b-5p was over-expressed in PTC patients with extrathyroidal invasion and cervical lymph node metastasis (Peng et al., 2014). Other than PTC, miR-199b-5p is also involved with the occurrence and development of leukemia (Flamant et al., 2010), liver cancer (Wang et al., 2011) and lung cancer (Nymark et al., 2011).

\section{miR-221/222}

The similar expression pattern between miR-221 and miR-222 can be explained by their location with both being clustered on chromosome X (Pallante et al., 2014). Many PTC studies investigating miRNAs expression identified miR-221/222 as the most consistently upregulated miRNAs and have significant association with clinicopathological features. PTC with LNM showed differential miR-222 expression as compared to PTC without LNM. A high serum miR-222 level has significant correlation with the presence of LNM (Yu et al., 2012). Enhanced expression of miRNA-221 and miRNA-222 was found in patients with cervical lymph node metastasis and advanced TNM stage (Sun et al., 2013). In a separate study, Lee and colleagues retrospectively recruited PTC patients with and without recurrence, which at the same time translated to patients with and without LNM based on the selection criteria. The authors demonstrated that the level of miR-222 in the tumor is associated with PTC recurrence; however, miR-221 showed no significant association with tumor recurrence (Lee et al., 2013). Higher expression levels of miR-222, together with miR221 , were detected in thyroid cancer patients with capsule invasion, vascular invasion and LNM when compared to the patients without these features (Acibucu et al., 2014).

Elevated expression of miR-221/222 has been observed in PTC relative to normal or benign thyroid diseases (Visone et al., 2007; Chen et al., 2008; Yu et al., 2012; Acibucu et al., 2014). Increased expression of miRNA-221 was evident in patients with tumor larger than $2 \mathrm{~cm}$ compared to smaller lesions (Sun et al., 2013). miR-221/222 are also potential markers for PTC aggressiveness as shown by two studies (Yip et al., 2011; Yang et al., 2013). Aggressive PTC is characterized by extrathyroidal extension, local recurrence, LNM and/or distant metastasis, hence their findings added further to the knowledge of possible roles of miR-221/222 in LNM. Moreover, miR-222, but not miR-221, was elevated in a highly invasive subpopulation of PTC cell lines by $8-10$ fold compared to the control group. These findings further strengthened the potential involvement of miR-221 and/ or miR-222 in modulating LNM in PTC.

Kim and colleagues utilized the microarray expression profiling technique and developed a bioluminescence imaging tool called the Gaussia luciferase (Gluc) reporter system in order to study genes regulated by miR-221 in PTC. In vitro analysis revealed thousands of genes regulated by miR-221 including $H O X B 5$ which was significantly downregulated. In addition, this miRNA modified the gene expression pattern of normal thyroid cells toward PTC. Furthermore, the authors demonstrated dose-dependent regulation of HOXB5 both in vitro and in vivo by endogenous or exogenous miR-221 (Kim et al., 2008). The proposed imaging system could be a useful tool for noninvasive in vivo long-term monitoring of functional modalities targeting of miR-221.

A homeobox (HOX) is a sequence of $\sim 180$ base pairs within genes that code for a protein domain called homeodomain (Stein et al., 1996). HOX genes are highly conserved and encode nuclear proteins that function as transcription factors (TF) during normal organogenesis (Gehring et al., 1986). The HOX gene family has also been intrinsic in human diseases particularly cancers. Aberrant expression of HOX genes has been reported for thyroid cancer, in both tissues and cell lines. A study by Takahashi and colleagues showed that HOXB1, HOXB9, $H O X D 10, H O X C 12$, and $H O X D 13$ were not expressed 
in normal thyroid tissues but were expressed in several anaplastic thyroid cancer cell lines. On the other hand, $H O X D 9$ was expressed only in thyroid cancer cell lines but was silenced in normal thyroid tissues. HOXB4 was the only HOX gene expressed in all thyroid cancer cell lines and normal thyroid tissues (Takahasi et al., 2004). Another study reported irregular expression of the HOX paralogous group 13 genes; HOXA13, HOXB13, HOXC13, and HOXD13 in well-differentiated thyroid cancers and their expression pattern was linked to the pathogenesis and differential diagnosis of thyroid cancers (Cantile et al., 2013). For instance, HOXA13 nuclear expression showed a significant and gradual increase from adenoma, to classical papillary carcinoma, follicular variant papillary carcinoma and follicular carcinoma (Cantile et al., 2013). HOXB13 exhibited the opposite trend of decreasing expression in the transition from non-neoplastic tissues to different tumour histologic types (Cantile et al., 2013).

One of the widely studied gene targets of miR-221/ miR-222 is $p 27^{\text {Kipl }}$. Enforced expression of miR-221/ miR-222 led to reduction of $p 27^{\text {Kip } 1}$ protein levels in the thyroid carcinoma cell line (TPC-1) and cervical adenocarcinoma cell line (HeLa) without significant changes in $p 27^{K i p l}$ mRNA levels. The forced expression also increased the transition from the G1 to the $\mathrm{S}$ phase in the thyroid papillary carcinoma cell line (Visone et al., 2007b). Overexpression of miR-221 increased the colony-forming ability of thyroid cancer cells, suggesting the role of miR-221 in thyroid carcinoma cell proliferation (Pallante et al., 2006).

High-mobility group box 1 protein (HMGB1) is a pro-inflammatory cytokine that is actively secreted by a diverse type of cells including macrophages, activated monocytes, dendritic cells, endothelial cells and certain cancer cells (Pikarsky et al., 2004). Mardente and colleagues demonstrated that HMG1 is overexpressed in thyroiditis and in PTC's microenvironment (Mardente et al., 2010). This cytokine was shown to influence miR221/222 expression. Addition of HMGB1 to the culture medium increases the expression of miR-221 by 3 -fold and miR-222 by 7-fold in both primary cultures of excised papillary lesions and in an established PTC cell line. The HMGB1-induced overexpression of oncogenic miR-221 and miR-222 is significantly associated with increased cell growth and motility (Mardente et al., 2012).

Despite being consistently reported to be upregulated in PTC with LNM, a study by Wang and colleagues (Wang et al., 2013) concluded that miR-221/222 is not associated with LNM. Another two studies reported significant association of only miR-222, but not miR-221, with LNM (Yu et al., 2012; Lee et al., 2013). These contradictory findings certainly warranted further profiling in larger samples and functional validation.

\section{miR-451}

miR-451 is highly conserved among vertebrates (Yang et al., 2010) and is located on chromosome 17 at position q11.2, 100 base pairs downstream of the miR-144 gene (Altuvia et al., 2005). Many studies have emphasized the roles of miR-451 in human cancers including in gastric cancer (Bandres et al., 2009), breast cancer (Bergamaschi and Katzenellenbogen, 2012), glioblastoma (Gal et al., 2008), and leukemia (Ju et al., 2009), demonstrating a critical function of miR-451 in carcinogenesis. In lung cancer, the downregulation of miR-451 was significantly correlated with LNM (Wang et al., 2011b). In a more recent study, re-expression of miR-451 could reverse epithelial-to-mesenchymal (EMT) to mesenchymalto-epithelial transition (MET) and inhibit invasion and metastasis of docetaxel-resistant lung adenocarcinoma cells both in vitro and in vivo (Chen et al., 2014). These findings suggest the role of miR-451 in the metastasis cascade.

In contradiction to the findings of miR-451 downregulation in various malignancies compared with adjacent noncancerous normal tissues, this miRNA was shown to be significantly upregulated in PTC with LNM as compared to PTC without LNM (Wang et al., 2013). Moreover, miR-451 upregulation in lateral lymph node (LLN) metastasis were significantly greater than those in central lymph node (CLN) metastasis (Wang et al., 2013). Unfortunately, this is the only study so far that has reported upregulation of miR-451 in PTC with LNM hence a functional study to validate the function of this miRNA in regulating LNM is certainly justified.

miR-1202

miR-1202 is located on chromosome 6 at position q25.3 in the human genome. It was shown to be significantly associated with LNM and is upregulated in PTC with LNM as compared to PTC without LNM (Wang et al., 2013). In addition, high expression of miR-1202 has been reported in adrenocortical carcinoma and is associated with poor survival (Ozata et al., 2011).

miR-2861

Investigation of miRNA deregulation in PTC with LNM led to the identification of miR-2861 upregulation in both the screening and validation cohort of samples (Wang et al., 2013). Together with miR-451, miR-2861 overexpression in LLN metastasis were greater than those in CLN metastasis (Wang et al., 2013). The literature regarding this miRNA in cancer is scarce; however, the involvement of miR-2186 has been reported in bone formation ( $\mathrm{Li}$ et al., 2009).

\section{miRNAs downregulated in PTC with LNM}

\section{Let-7 family}

Let-7 is highly conserved across diverse animal species (Pasquinelli et al., 2000). The deregulation of let-7 has been shown in many types of cancer with its function in regulating cell proliferation and differentiation during development in various species (Boyerinas et al., 2010). Regarded as a tumor suppressive miRNA, let-7 is involved in the regulation self-renewal and tumorigenicity of breast cancer cells (Yu et al., 2007). It also acts as a potential growth suppressor in human colon cancer cells (Akao et al., 2006) and its reduced expression is associated with shortened postoperative survival in human lung cancers.

Several groups reported the downregulation of let7 in PTC as well as all thyroid cancers of follicular 
origin (Pallante et al., 2006; Visone et al., 2007a; Braun and Hüttelmaier, 2011). On the contrary, in a study on circulating miRNA profiles in patients with PTC or benign nodules and healthy controls, the expression of serum let-7e was significantly increased in PTC cases relative to other groups (Yu et al., 2012). In addition, further stratification of the PTCs according to lymph node status revealed that serum let-7e was downregulated in PTC with LNM in comparison to PTC without LNM (Yu et al., 2012). Taken together, these findings implied that the exact role of let-7 in tissues and circulation in PTCs warrant further investigation.

To gain insight into the alterations in miRNA expression that might regulate lymphatic metastasis of PTC, Gao and colleagues established highly metastatic PTC cell lines, namely IHH-4M-1, IHH-4M-2 and IHH-4M-3, from a clonal cell line of human PTC with cervical lymph node metastasis (denoted as IHH-4) and performed miRNA microarray analysis. One of the let-7 families, let-7b, was upregulated in highly invasive cells by 17 - 25 fold (Gao et al., 2010). This finding suggested the involvement of let-7 in regulating metastasis and malignant transformation.

One of the key genetic events frequently associated to PTC is RET/PTC rearrangement, which resulted in enhanced proliferation and dedifferentiation by the activation of the RET/PTC-RAS-BRAF-mitogenactivated protein kinase (MAPK) pathway (Nikiforov and Nikiforova, 2011; Nikiforov, 2011; Xing, 2013). Ricarte-Filho and colleagues reported that oncogenic activation of RET/PTC3 in PCCL3 rat thyroid cells significantly reduces let-7f expression (Ricarte-Filho et al., 2009). Furthermore, forced expression of let-7 microRNA in TPC-1 cells, which harbor RET/PTC1 rearrangement, resulted in inhibition of MAPK activation and consequently led to reduction of TPC-1 cell growth (Ricarte-Filho et al., 2009). Decreased expression of cell cycle stimulators such as MYC and CCND1 (cyclin D1), increased the cell cycle inhibitor P21 and enhanced the transcriptional expression of molecular markers of thyroid differentiation such as TITF1 and TG (Ricarte-Filho et al., 2009). These findings pointed to a model that reduced expression of let-7f might be a crucial molecular event in RET/PTC malignant transformation (Ricarte-Filho et al., 2009) and this was the first functional demonstration of an association of let-7 in thyroid cancer cell growth and differentiation.

miR-16

Downregulation of miR-16 expression is evident in the invasive subpopulation of a thyroid cancer cell line (Gao et al., 2010). miR-16 is also underexpressed in aggressive PTC (Yang et al., 2013). Integrated analysis revealed that the expression of FN1 and ITGA2 was upregulated with the downregulation of miR-16 (Yang et al., 2013). Furthermore, a luciferase reporter assay confirmed the direct interaction of miR-16 with FN1 and ITGA2. These genes are related to the extracellular matrix (ECM) or signal transduction pathways linked to the aggressiveness of tumors. Collectively, the authors proposed that interaction of miR-16 with FN1 and
ITGA2 might contribute to the aggressiveness of PTCs by regulating the invasion and migration processes (Yang et al., 2013).

miR-513

miR-513 is a primate-specific miRNA subfamily located on $\mathrm{X}$ chromosome that has undergone rapid evolution (Sun et al., 2013). Evidence suggests that this subfamily is favorably expressed in the testis; however, the functional importance of this miRNA subfamily has remained unknown. miR-513 has been reported to be downregulated in highly invasive PTC when compared to less invasive PTC (Peng et al., 2014). Different roles of miR-513 has been reported in gastric cancer, whereby for example forced expression of miR-513b inhibits cell proliferation, migration and promotes apoptosis by targeting high mobility group-box 3 protein (Chen et al., 2014b).

miR-542

Similar to miR-513, miR-542 is also located on the human X chromosome. Wang and colleagues reported that miR-542-5p was downregulated in LNM when compared with non-LNM group in PTC (Wang et al., 2013). miR542 downregulation was also reported in conventional FTC compared with oncocytic FTC group (Dettmer et al., 2013).

\section{miR-564}

Located on chromosome $3 \mathrm{p} 21.31$, upregulation of miR-564 was reported in a study comparing PTC and benign thyroid tissues samples (Vriens et al., 2012). This is in contrast with another study, whereby miR-564 was downregulated in PTC samples with LNM (Wang et al., 2013). A similar finding was also observed in chronic myeloid leukemia (CML). Rokah and colleagues showed miR-564 downregulation in CML cell lines and patients in comparison to non-CML cell lines and healthy blood cells (Rokah et al., 2012).

\section{miR-613, miR-664 and miR-1243}

miR-613 and miR-664* were downregulated in LNM cases when compared with cases without LNM (Wang et al., 2013; Yang et al., 2013). miR-1243 was downregulated in the highly invasive PTC compared to those which were less invasive (Peng et al., 2014). There is only a single publication demonstrating downregulation of these miRNAs and their functional characterization is still lacking. Hence, more supporting literature is required to validate their role in LNM in PTC.

\section{miRNAs Potentially Involved in Lymph Node Metastasis}

$\operatorname{miR}-15$

The miR-15 family includes miR-15a and miR-15b, as well as miR-16-1, miR-16-2, miR-195 and miR-497. In humans, miR-15a is located at chromosome position $13 q 14$ and is clustered with miR-16 within 0.5 kilobases (Lagos-Quintana et al., 2001). Studies have shown that miR-15a functions as a tumor suppressor with the oncogene BCL2 as its target (Bonci et al., 2008). 
miR-15a is among the first miRNAs identified in PTC. The investigation of miRNA expression in two human PTC cell lines bearing a RET mutation and in normal thyroid cell lines revealed a downregulation of miR-15a (Cahill et al., 2006). This miRNA was also shown to play an important role in tumor cell metastasis progression or EMT process (Visone et al., 2007).

$m i R-26$

The miR-26 family comprises of three members, namely miR-26a-1, miR-26a-2 and miR-26b, which are located in chromosomes 3,12 and 2 respectively. The mature miRNA of miR-26a-1 and miR-26a-2 possess the same sequence with the exception of two different nucleotides in the mature miR-26b. The miR-26 seed region, which is highly consistent in members of different genera, is an important region for binding to target mRNAs (Gao et al., 2012).

The miRNA expression profiles were analysed in anaplastic thyroid carcinoma (ATC) samples compared to normal thyroid tissues and significant downregulation of several miRNAs including miR-26a was observed (Visone et al., 2007; Mitomo et al., 2008). Integrated miRNA-microarray analysis revealed downregulation of miR-26a in PTC (He et al., 2005). It was reported that miR-26a suppresses proliferation and colony formation efficiency, induces G2 cell cycle arrest, promotes cell apoptosis and inhibits tumor growth in vivo by targeting CKS2 (Lv et al., 2013).

miR-29

The human miR-29 family of microRNAs has three mature members which are miR-29a, miR-29b, and miR-29c. miR-29b-1 and miR-29b-2 have identical mature sequences, and together are called miR-29b. miR-29 members are encoded by two gene clusters. Binding sites for several transcriptional factors have been identified in the promoter regions of miR-29 genes. This miRNA family directly targets at least 16 extracellular matrix genes, providing a dramatic example of a single microRNA targeting a large group of functionally related genes (Kriegel et al., 2012). There is only a single study reporting miR-29 profile in PTC. miR-29 downregulation was described in the invasive subpopulation compared to control subpopulation in human PTC cell line (Gao et al., 2010).

miR-34

miR-34 is a key regulator of tumor suppression. The miR-34 precursor family comprises of three major mature miRNAs which are miR-34a, miR-34b and miR$34 \mathrm{c}$. These three miR-34 precursors are produced by two different transcription units. miR-34a is produced by its own transcript whereas miR-34b and miR-34c are produced by a common primary transcript (Misso et al., 2014). There are several reports which showed members of the miR-34 family directly targeting the tumor suppressor gene TP53 and their upregulation induces apoptosis and cell-cycle arrest (Chang et al., 2007; Corney et al., 2007; He et al., 2007).

The first member of the family, miR-34a, is a transcriptional target for TP53 and is frequently inactivated in various types of human cancers including PTC. It was reported that miR-34a acts as a tumor suppressor in several types of cancer through repression of a group of genes that promote cell proliferation (Mackiewicz et al., 2011; Wang et al., 2013; Zhao et al., 2013). However, miR-34a has been reported to be upregulated in PTC tissues and cell lines (Cahill et al., 2006; Tetzlaff et al., 2007; Sheu et al., 2010; Marini et al., 2011; Ma et al., 2013). In contrast, another two family members of miR-34, namely miR-34b and miR-34c, are reported to be downregulated in PTC (Cahill et al., 2006; arini et al., 2011; Yip et al., 2011). In addition, downregulation of miR-34b was shown to be associated with the aggressive PTC subtype by targeting MET (Yip et al., 2011). The finding was further strengthened by another report that demonstrated the negative regulation of MET by miR-34b and miR-34c (Migliore et al., 2008).

\section{Concluding Remarks}

It is well known that PTC is a rather indolent tumor with a low mortality rate and good prognosis; however, patients with LNM, which often occur in PTC, have a poorer prognosis, especially in those older than 45 years of age. Lateral neck dissection could provide precise tumor staging concerning the nodal status and diminish the risk of LNM recurrence; however the operation itself also increases the chance of complications after surgery. Furthermore, prophylactic lateral neck dissection in PTC patients with clinically negative LNM has been highly controversial.

Both neck ultrasonography and computed tomography have limited value for the prediction of LNM; hence it is crucial to find novel biomarkers that can be used for this purpose and also as a standard for optimizing therapy and long-term follow-up care. As discussed in this mini review, several miRNAs have been shown to be dysregulated in PTC with and without LNM. However, functional studies of these miRNAs are still lacking. These findings point toward the potential application of miRNAs as biomarkers for predicting LNM and also a prerequisite for in-depth research to determine the mechanisms of how these miRNAs may govern lymph node metastasis in PTC.

However, there are several challenges that await such research. The study of in vitro and in vivo miRNA function is complicated by the fact that manipulation of a single miRNA may not lead to significant consequences. The presence of miRNA families, which are defined as a group of miRNAs with the same seed region, raises the question as to whether researchers should investigate all the family members or just a particular miRNA. In addition, due to the non-canonical and partially complementary binding properties of miRNA, nearly half of the miRNA targets contain binding sites for at least two miRNAs. Therefore, miRNAs binding to the same target can synergize and/ or antagonize the expression of target genes, obscuring studies on single miRNA.

Numerous studies have evidently established a role for miRNAs in regulation of lymph node metastasis in other malignancies. The advancement of research technologies such as next generation sequencing has enabled the 
identification of thousands of miRNAs; however only several of these miRNAs have been clearly proven to be associated with lymph node metastasis. Despite rapid and abundant studies on expression profiling, the roles of the majority of the miRNAs in PTC remain unknown and therefore necessitates further exploration in vitro as well as in vivo.

\section{Acknowledgements}

This manuscript was supported by Fundamental Research Grant Scheme (FRGS) from the Ministry of Education Malaysia (FRGS/1/2014/SKK01/UKM/03/1).

\section{References}

Acibucu F, Dokmetas HS, Tutar Y, et al (2014). Correlations between the expression levels of micro-RNA146b, 221, 222 and $p 27^{\text {Kipl }}$ protein mRNA and the clinicopathologic parameters in papillary thyroid cancers. Exp Clin Endocrinol Diabetes, 122, 137-43.

Akao Y, Nakagawa Y, Naoe T (2006). Let-7 microRNA functions as a potential growth suppressor in human colon cancer cells. Biol Pharm Bull, 29, 903-6.

Altuvia Y, Landgraf P, Lithwick G, et al (2005). Clustering and conservation patterns of human microRNAs. Nucleic Acids Res, 33, 2697-706.

Bandres E, Bitarte N, Arias F, et al (2009). MicroRNA-451 regulates macrophage migration inhibitory factor production and proliferation of gastrointestinal cancer cells. Clin Cancer Res, 15, 2281-90.

Bartel DP (2009). MicroRNAs: target recognition and regulatory functions. Cell, 136, 215-233.

Bergamaschi A, Katzenellenbogen BS (2012). Tamoxifen downregulation of miR-451 increases 14-3-3zeta and promotes breast cancer cell survival and endocrine resistance. Oncogene, 31, 39-47.

Bonci D, Coppola V, Musumeci M, et al (2008). The miR15a-miR-16-1 cluster controls prostate cancer by targeting multiple oncogenic activities. Nat Med, 14, 1271-7.

Boyerinas B, Park SM, Hau A, et al (2010). The role of let-7 in cell differentiation and cancer. Endocr Relat Cancer, 17, 19-36.

Braun J, Hüttelmaier S (2011). Pathogenic mechanisms of deregulated microRNA expression in thyroid carcinomas of follicular origin. Thyroid Res, $\mathbf{4}, 1$.

Cahill S, Smyth P, Finn SP, et al (2006). Effect of ret/PTC 1 rearrangement on transcription and post-transcriptional regulation in a papillary thyroid carcinoma model. $\mathrm{Mol}$ Cancer, 11, 70 .

Calin GA, Sevignani C, Dumitru CD, et al (2004). Human microRNA genes are frequently located at fragile sites and genomic regions involved in cancers. Proc Natl Acad Sci $U$ $S$ A, 101, 2999-3004.

Cancer Genome Atlas Research Network (2014). Integrated genomic characterization of papillary thyroid carcinoma. Cell, 159, 676-90.

Cantile M, Scognamiglio G, La Sala L, et al (2013). Aberrant expression of posterior HOX genes in well differentiated histotypes of thyroid cancers. Int J Mol Sci, 14, 21727-40.

Chang TC, Wentzel EA, Kent OA, et al (2007). Transactivation of miR-34a by 533 broadly influences gene expression and promotes apoptosis. Mol Cell, 26, 745-52.

Chen D, Huang J, Zhang K, et al (2014). MicroRNA-451 induces epithelial-mesenchymal transition in docetaxel-resistant lung adenocarcinoma cells by targeting proto-oncogene c-Myc.
Eur J Cancer, 50, 3050-67.

Chen X, Zhao G, Wang F, et al (2014). Upregulation of miR513b inhibits cell proliferation, migration, and promotes apoptosis by targeting high mobility group-box 3 protein in gastric cancer. Tumour Biol, 35, 11081-9.

Chen YT, Kitabayashi N, Zhou XK, et al (2008). MicroRNA analysis as a potential diagnostic tool for papillary thyroid carcinoma. Mod Pathol, 21, 1139-46.

Chou CK, Chen RF, Chou FF, et al (2010). miR-146b is highly expressed in adult papillary thyroid carcinomas with high risk features including extrathyroidal invasion and the BRAF(V600E) mutation. Thyroid, 20, 489-94.

Chou CK, Yang KD, Chou FF, et al (2013). Prognostic implications of miR-146b expression and its functional role in papillary thyroid carcinoma. J Clin Endocrinol Metab, 98, 196-205.

Cognetti OM, Pribitkin EA, Keane WB (2008). Management of the neck in differentiated thyroid cancer. Surg Oneal Clin N Am, 17, 157-73.

Corney DC, Flesken-Nikitin A, Godwin AK, et al (2007). MicroRNA-34b and MicroRNA-34c are targets of p53 and cooperate in control of cell proliferation and adhesionindependent growth. Cancer Res, 67, 8433-8.

de la Chapelle A, Jazdzewski K (2011). MicroRNAs in thyroid cancer. J Clin Endocrinol Metab, 96, 3326-36.

Deng X, Wu B, Xiao K, et al (2015). MiR-146b-5p promotes metastasis and induces epithelial-mesenchymal transition in thyroid cancer by targeting ZNRF3. Cell Physiol Biochem, 35, 71-82.

Dettmer M, Vogetseder A, Durso MB, et al (2013). MicroRNA expression array identifies novel diagnostic markers for conventional and oncocytic follicular thyroid carcinomas. $J$ Clin Endocrinol Metab, 98, 1-7.

Ding J, Huang S, Wu S, et al (2010). Gain of miR-151 on chromosome 8q24.3 facilitates tumour cell migration and spreading through downregulating RhoGDIA. Nat Cell Biol, 12, 390-9.

Fassina A, Cappellesso R, Simonato F, et al (2014). A 4-MicroRNA signature can discriminate primary lymphomas from anaplastic carcinomas in thyroid cytology smears. Cancer Cytopathol, 122, 274-81.

Felekkis K, Touvana E, Stefanou Ch, et al (2010). microRNAs: a newly described class of encoded molecules that play a role in health and disease. Hippokratia, 14, 236-40.

Flamant S, Ritchie W, Guilhot J, et al (2010). Micro-RNA response to imatinib mesylate in patients with chronic myeloid leukemia. Haematologica, 95, 1325-33.

Gal H, Pandi G, Kanner AA, et al (2008). miR-451 and Imatinib mesylate inhibit tumor growth of Glioblastoma stem cells. Biochem Biophys Res Commun, 376, 86-90.

Gao J, Liu QG (2012). The role of miR-26 in tumors and normal tissues (Review). Oncol Lett, 6, 1019-23.

Gao Y, Wang C, Shan Z, et al (2010). miRNA expression in a human papillary thyroid carcinoma cell line varies with invasiveness. Endocr J, 57, 81-6.

Gehring WJ, Hiromi Y (1986). Homeotic genes and the homeobox. Annu Rev Genet, 20, 147-73.

Geraldo MV, Yamashita AS, Kimura ET (2012). MicroRNA miR-146b-5p regulates signal transduction of TGF- $\beta$ by repressing SMAD4 in thyroid cancer. Oncogene, 31, 191022.

Guerrero MA, Clark OH (2011). Controversies in the Management of Papillary Thyroid Cancer Revisited. ISRN Oncol, 2011, 303128,

Hao HX, Xie Y, Zhang Y, et al (2012). ZNRF3 promotes Wnt receptor turnover in an R-spondin-sensitive manner. Nature, 485, 195-200. 
He H, Jazdzewski K, Li W, et al (2005). The role of microRNA genes in papillary thyroid carcinoma. Proc Natl Acad Sci U $S$ A, 102, 19075-80.

He L, He X, Lim LP, et al (2007). A microRNA component of the p53 tumour suppressor network. Nature, 447, 1130-4.

Hotomi M, Sugitani I, Toda K, et al (2012). A novel definition of extrathyroidal invasion for patients with papillary thyroid carcinoma for predicting prognosis. World J Surg, 36, $1231-40$

Hsieh SH, Chen ST, Hsueh C, et al (2012). Gender-specific variation in the prognosis of papillary thyroid cancer TNM stages II to IV. Int J Endocrinol, 2012, 379097.

Huang KH, Lan YT, Fang WL, et al (2015). The correlation between miRNA and lymph node metastasis in gastric cancer. Biomed Res Int, 2015, 543163.

Iorio MV, Croce CM (2012). MicroRNA dysregulation in cancer: diagnostics, monitoring and therapeutics. A comprehensive review. EMBO Mol Med, 4, 143-59.

Ito Y, Kudo T, Kobayashi K, et al (2012). Prognostic factors for recurrence of papillary thyroid carcinoma in the lymph nodes, lung, and bone: analysis of 5,768 patients with average 10-year follow-up. World J Surg, 36, 1274-8.

Jonklaas J, Nogueras-Gonzalez G, Munsell M, et al (2012). The impact of age and gender on papillary thyroid cancer survival. J Clin Endocrinol Metab, 97, 878-87.

Ju X, Li D, Shi Q, et al (2009). Differential microRNA expression in childhood B-cell precursor acute lymphoblastic leukemia. Pediatr Hematol Oncol, 26, 1-10.

Kim HJ, Kim YH, Lee DS, et al (2008). In vivo imaging of functional targeting of miR-221 in papillary thyroid carcinoma. J Nucl Med, 49, 1686-93.

Kozomara A, Griffiths-Jones S (2014). miRBase: annotating high confidence microRNAs using deep sequencing data. Nucleic Acids Res, 42, 68-73.

Kramer JA, Schmid KW, Dralle H, et al (2010). Primary tumour size is a prognostic parameter in patients suffering from differentiated thyroid carcinoma with extrathyroidal growth: results of the MSDS trial. Eur J Endocrinol, 163, 637-44.

Krell J, Frampton AE, Jacob J, et al (2012). The clinicopathologic role of microRNAs miR-9 and miR-151-5p in breast cancer metastasis. Mol Diagn Ther, 16, 167-72.

Kriegel AJ, Liu Y, Fang Y, et al (2012). The miR-29 family: genomics, cell biology, and relevance to renal and cardiovascular injury. Physiol Genomics, 44, 237-44.

Lagos-Quintana M, Rauhut R, Lendeckel W, et al (2001). Identification of novel genes coding for small expressed RNAs. Science, 294, 853-58.

Lee DY, Jeyapalan Z, Fang L, et al (2010). Expression of versican 3 '-untranslated region modulates endogenous microRNA functions. PLoS One, 5, 13599.

Lee J, Song Y, Soh EY (2014). Central lymph node metastasis is an important prognostic factor in patients with papillary thyroid microcarcinoma. J Korean Med Sci, 29, 48-52.

Lee JC, Zhao JT, Clifton-Bligh RJ, et al (2013). MicroRNA-222 and microRNA-146b are tissue and circulating biomarkers of recurrent papillary thyroid cancer. Cancer, 119, 4358-65.

Leonardi GC, Candido S, Carbone M, et al (2012). microRNAs and thyroid cancer: biological and clinical significance (Review). Int J Mol Med, 30, 991-9.

Li H, Xie H, Liu W, et al (2009). A novel microRNA targeting HDAC5 regulates osteoblast differentiation in mice and contributes to primary osteoporosis in humans. J Clin Invest, 119, 3666-77.

Li X, Abdel-Mageed AB, Mondal D, et al (2013). MicroRNA expression profiles in differentiated thyroid cancer, a review. Int J Clin Exp Med, 6, 74-80.

Liu X, Sempere LF, Ouyang H, et al (2010). MicroRNA-31 functions as an oncogenic microRNA in mouse and human lung cancer cells by repressing specific tumor suppressors. J Clin Invest, 120, 1298-309.

Lodewijk L, Prins AM, Kist JW, et al (2012). The value of miRNA in diagnosing thyroid cancer: a systematic review. Cancer Biomark, 11, 229-38.

Lundgren CI, Hall P, Dickman PW, et al (2006). Clinically significant prognostic factors for differentiated thyroid carcinoma: a population-based, nested case-control study. Cancer, 106, 524-31.

Lv M, Zhang X, Li M, et al (2013). miR-26a and its target CKS2 modulate cell growth and tumorigenesis of papillary thyroid carcinoma. PLoS One, 8, 67591.

Ma Y, Qin H, Cui Y (2013). MiR-34a targets GAS1 to promote cell proliferation and inhibit apoptosis in papillary thyroid carcinoma via PI3K/Akt/Bad pathway. Biochem Biophys Res Commun, 441, 958-63.

Machens A, Hinze R, Thomusch O, et al (2002). Pattern of nodal metastasis for primary and reoperative thyroid cancer. World J Surg, 26, 22-8.

Mackiewicz M, Huppi K, Pitt JJ, et al (2011). Identification of the receptor tyrosine kinase AXL in breast cancer as a target for the human miR-34a microRNA. Breast Cancer Res Treat, 130, 663-79.

Mardente S, Mari E, Consorti F, et al (2012). HMGB1 induces the overexpression of miR-222 and miR-221 and increases growth and motility in papillary thyroid cancer cells. Oncol Rep, 28, 2285-9.

Mardente S, Zicari A, Consorti F, et al (2010). Cross-talk between NO and HMGB1 in lymphocytic thyroiditis and papillary thyroid cancer. Oncol Rep, 24, 1455-61.

Marini F, Luzi E, Brandi ML (2011). MicroRNA Role in Thyroid Cancer Development. J Thyroid Res, 2011, 407123.

Mattick JS (2001). Non-coding RNAs: the architects of eukaryotic complexity. EMBO Rep, 2, 986-91.

McConahey WM, Hay ID, Woolner LB, et al (1986). Papillary thyroid cancer treated at the mayo clinic, 1946 through 1970: initial manifestations, pathologic findings, therapy, and outcome. Mayo Clin Proc, 61, 978-96.

Migliore C, Petrelli A, Ghiso E, et al (2008). MicroRNAs impair MET-mediated invasive growth. Cancer Res, 68, 10128-36.

Misso G, Di Martino MT, De Rosa G, et al (2014). miR-34: a new weapon against cancer? Mol Ther Nucleic Acids, 3, 194.

MitomoS, Maesawa C, Ogasawara S, et al (2008). Downregulation of miR-138 is associated with overexpression of human telomerase reverse transcriptase protein in human anaplastic thyroid carcinoma cell lines. Cancer Sci, 99, 280-6.

Moo TA, Fahey TJ 3rd (2011). Lymph node dissection in papillary thyroid carcinoma. Semin Nucl Med, 41, 84-8.

Nam S, Li M, Choi K, et al (2009). MicroRNA and mRNA integrated analysis (MMIA): a web tool for examining biological functions of microRNA expression. Nucleic Acids Res, 37, 356-62.

Nikiforov YE (2011). Molecular analysis of thyroid tumors. Mod Pathol, 24, 34-43.

Nikiforov YE, Nikiforova MN (2011). Molecular genetics and diagnosis of thyroid cancer. Nat Rev Endocrinol, 7, 569-80.

Nikiforova MN, Tseng GC, Steward D, et al (2008). MicroRNA expression profiling of thyroid tumors: biological significance and diagnostic utility. J Clin Endocrinol Metab, 93, 1600-8.

Nymark P, Guled M, Borze I, et al (2011). Integrative analysis of microRNA, mRNA and aCGH data reveals asbestosand histology-related changes in lung cancer. Genes Chromosomes Cancer, 50, 585-97.

Ozata DM, Caramuta S, Velazquez-Fernandez D, et al (2011). The role of microRNA deregulation in the pathogenesis of adrenocortical carcinoma. Endocr Relat Cancer, 27, 643-55. 
Pallante P, Battista S, Pierantoni GM, et al (2014). Deregulation of microRNA expression in thyroid neoplasias. Nat Rev Endocrinol, 10, 88-101.

Pallante P, Visone R, Croce CM, et al (2010). Deregulation of microRNA expression in follicular-cell-derived human thyroid carcinomas. Endocr Relat Cancer, 17, 91-104.

Pallante P, Visone R, Ferracin M, et al (2006). MicroRNA deregulation in human thyroid papillary carcinomas. Endocr Relat Cancer, 13, 497-508.

Pasquinelli AE, Reinhart BJ, Slack F, et al (2000). Conservation of the sequence and temporal expression of let-7 heterochronic regulatory RNA. Nature, 408, 86-9.

Peng Y, Li C, Luo DC, et al (2014). Expression profile and clinical significance of microRNAs in papillary thyroid carcinoma. Molecules, 19, 11586-99.

Pikarsky E, Porat RM, Stein I, et al (2004). NF- «B funtions as a tumor promoter in inflammation-associated cancer. Nature, 431, 261-66.

Qu N, Zhang L, Ji QH, et al (2014). Number of tumor foci predicts prognosis in papillary thyroid cancer. BMC Cancer, 4, 914 .

Ricarte-Filho JC, Fuziwara CS, Yamashita AS, et al (2009). Effects of let-7 microRNA on cell growth and differentiation of papillary thyroid cancer. Transl Oncol, 2, 236-41.

Rokah OH, Granot G, OvcharenkoA, et al (2012). Downregulation of miR-31, miR-155, and miR-564 in chronic myeloid leukemia cells. PLoS One, 7, 35501.

Rossing M, Borup R, Henao R, et al (2012). Down-regulation of microRNAs controlling tumourigenic factors in follicular thyroid carcinoma. J Mol Endocrinol, 48, 11-23.

Samimi H,Zaki Dizaji M, Ghadami M, et al (2013). MicroRNAs networks in thyroid cancers: focus on miRNAs related to the fascin. J Diabetes Metab Disord, 12, 31.

Scheumann GF, Gimm O, Wegener G, et al (1994). Prognostic significance and surgical management of locoregional lymph node metastases in papillary thyroid cancer. World J Surg, $18,559-67$.

Shaha AR, Shah JP, Loree TR (1996). Patterns of nodal and distant metastasis based on histologic varieties in differentiated carcinoma of the thyroid. Am J Surg, 172, 692-4.

Wada N, Duh QY, Sugino K, et al (2003). Lymph node metastasis from 259 papillary thyroid microcarcinomas: frequency, pattern of occurrence and recurrence, and optimal strategy for neck dissection. Ann Surg, 237, 399-407.

Shen S, Yue H, Li Y, et al (2014). Upregulation of miR-136 in human non-small cell lung cancer cells promotes Erk1/2 activation by targeting PPP2R2A. Tumour Biol, 35, 631-40.

Sheu SY, Grabellus F, Schwertheim S, et al (2010). Differential miRNA expression profiles in variants of papillary thyroid carcinoma and encapsulated follicular thyroid tumours. $\mathrm{Br}$ J Cancer, 102, 376-82.

Slaby O, Svoboda M, Fabian P, et al (2007). Altered expression of miR-21, miR-31, miR-143 and miR-145 is related to clinicopathologic features of colorectal cancer. Oncol, 72, 397-402.

Stein S, Fritsch R, Lemaire L, et al (1996). Checklist: vertebrate homeobox genes. Mech Dev, 55, 91-108.

Sun Y, Yu S, Liu Y, et al (2013). Expression of miRNAs in papillary thyroid carcinomas is associated with BRAF mutation and clinicopathological features in Chinese patients. Int J Endocrinol, 2013, 128735.

Sun Z, Zhang Y, Zhang R, et al (2013). Functional divergence of the rapidly evolving miR-513 subfamily in primates. $B M C$ Evol Biol, 13, 255.

Takahashi Y, Hamada J, Murakawa K, et al (2004). Expression profiles of $39 \mathrm{HOX}$ genes in normal human adult organs and anaplastic thyroid cancer cell lines by quantitative real-time RT-PCR system. Exp Cell Res, 293, 144-53.

Tetzlaff MT, Liu A, Xu X, et al (2007). Differential expression of miRNAs in papillary thyroid carcinoma compared to multinodular goiter using formalin fixed paraffin embedded tissues. Endocr Pathol, 18, 163-73.

Vigneri R, Malandrino P, Vigneri P (2015). The changing epidemiology of thyroid cancer: why is incidence increasing? Curr Opin Oncol, 27, 1-7.

Visone R, Pallante P, Vecchione A, et al (2007). Specific microRNAs are downregulated in human thyroid anaplastic carcinomas. Oncogene, 26, 7590-5.

Visone R, Russo L, Pallante P, et al (2007). MicroRNAs (miR)221 and miR-222, both overexpressed in human thyroid papillary carcinomas, regulate 27Kip1 protein levels and cell cycle. Endocr Relat Cancer, 14, 791-8.

Vriens MR, Weng J, Suh I, et al (2012). Microrna expression profiling is a potential diagnostic tool for thyroid cancer. Cancer, 118, 3426-32.

Wang C, Song B, Song W, et al (2011). Underexpressed microRNA-199b-5p targets Hypoxia-Inducible Factor$1 \alpha$ in hepatocellular carcinoma and predicts prognosis of hepatocellular carcinoma patients. J Gastroenterol Hepatol, 26, 1630-7.

Wang R, Wang ZX, Yang JS, et al (2011). MicroRNA-451 functions as a tumor suppressor in human non-small cell lung cancer by targeting ras-related protein 14 (RAB14). Oncogene, 30, 2644-58.

Wang W, Li T, Han G, et al (2013). Expression and Role of miR34a in bladder cancer. Indian J Biochem Biophys, 50, 87-92.

Wang Z, Zhang H, He L, et al (2013). Association between the expression of four upregulated miRNAs and extrathyroidal invasion in papillary thyroid carcinoma. Onco Targets Ther, 6, 281-7.

Wang Z, Zhang H, Zhang P, et al (2013). Upregulation of miR2861 and miR-451 expression in papillary thyroid carcinoma with lymph node metastasis. Med Oncol, 30, 577.

Wojtas B, Ferraz C, Stokowy T, et al (2014). Differential miRNA expression defines migration and reduced apoptosis in follicular thyroid carcinomas. Mol Cell Endocrinol, 388, 1-9.

Xing M (2013). Molecular pathogenesis and mechanisms of thyroid cancer. Nat Rev Cancer, 13, 184-99.

Xing M, Alzahrani AS, Carson KA, et al (2013). Association between BRAF V600E mutation and mortality in patients with papillary thyroid cancer. JAMA, 309, 1493-501.

Yan LX, Huang XF, Shao Q, et al (2008). MicroRNA miR-21 overexpression in human breast cancer is associated with advanced clinical stage, lymph node metastasis and patient poor prognosis. $R N A, \mathbf{1 4}, 2348-60$.

Yang JS, Maurin T, Robine N, et al (2010). Conserved vertebrate miR-451 provides a platform for Dicer-independent, Ago2mediated microRNA biogenesis. Proc Natl Acad Sci U S A, 107, 15163-8.

Yang Z, Yuan Z, Fan Y, et al (2013). Integrated analyses of microRNA and mRNA expression profiles in aggressive papillary thyroid carcinoma. Mol Med Rep, 8, 1353-8.

Yip L, Kelly L, Shuai Y, et al (2011). MicroRNA signature distinguishes the degree of aggressiveness of papillary thyroid carcinoma. Ann Surg Oncol, 18, 2035-41.

Yu F, Yao H, Zhu P, et al (2007). let-7 regulates self renewal and tumorigenicity of breast cancer cells. Cell, 131, 1109-23.

Yu J, Wang F, Yang GH, et al (2006). Human microRNA clusters: genomic organization and expression profile in leukemia cell lines. Biochem Biophys Res Commun, 349, 59-68.

Yu S, Liu Y, Wang J, et al (2012). Circulating microRNA profiles as potential biomarkers for diagnosis of papillary thyroid carcinoma. J Clin Endocrinol Metab, 97, 2084-92. 
Yuan ZM, Yang ZL, Zheng Q (2014). Deregulation of microRNA expression in thyroid tumors. $J$ Zhejiang Univ Sci B, 15, 212-24.

Zaydfudim V, Feurer ID, Griffin MR, et al (2008). The impact of lymph node involvement on survival in patients with papillary and follicular thyroid carcinoma. Surgery, $\mathbf{1 4 4}$ 1070-8.

Zhao H, Ma B, Wang Y, et al (2013). miR-34a inhibits the metastasis of osteosarcoma cells by repressing the expression of CD44. PLoS One, 9, 78644. 